\section{Rotation of Chain Molecules in Solid Paraffins}

IT is found that the thermal expansion of the crystal lattice of $n$-paraffins is not equal in all directions. ${ }^{1}$ The increase in length of the $a$ axis measured in the range of liquid air temperature to room temperature is about 7 per cent, of the $b$ axis about 2 per cent, whereas the $c$ axis remains practically unaltered. This axis is parallel to the chain axes of the molecules.

Recent X-ray measurements on a series of solid paraffins between room temperature and the melting points show that the $a$ axes continue to increase at a higher rate than the $b$ axes. The result is a change in the lattice dimensions which will be discussed presently. Fig. 1 (i) illustrates the packing of the molecules characteristic of all the members of the scries. This arrangement is observed when the substances are at room temperature. Fig. 1 (ii) is the corresponding pattern near the melting points.

The rectangles represent the boundaries of the crosssection through the unit cell containing the $a$ and $b$ axes. The long axis of the chain molecule is perpendicular to the plane of the paper. The projections of the-chains, which consist of a zigzag arrangement

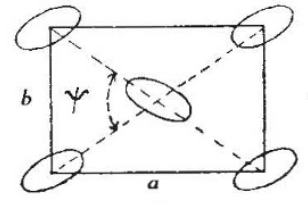

(i)

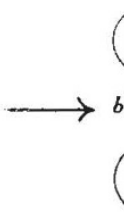

FIG. 1.

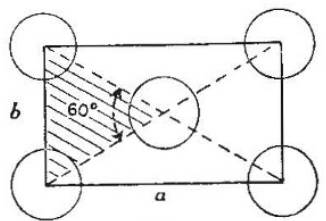

(ii) of $\mathrm{CH}_{2}$-groups, are shown as ellipses in Fig. 1 (i) There are two types of orientations present of these ellipses when the crystals are at room temperature. The angle $\psi$ is between $67^{\circ} 10^{\prime}$ and $67^{\circ} 40^{\prime}$ at $18^{\circ}-20^{\circ} \mathrm{C}$.

The angle $\psi$ depends upon the temperature. It decreases with rising temperature, the expansion of $a$ being larger than that of $b$, until it reaches $60^{\circ}$ near the melting point. This is found to happen with $\mathrm{C}_{21}, \mathrm{C}_{23}, \mathrm{C}_{24}, \mathrm{C}_{25}, \mathrm{C}_{26}, \mathrm{C}_{27}, \mathrm{C}_{29}, \mathrm{C}_{30}, \mathrm{C}_{31}, \mathrm{C}_{34}, \mathrm{H}_{44}$ and can, therefore, be regarded as the general behaviour of the whole series within that range. If $\psi=60^{\circ}$, the triangle shown by cross-hatchings is equilateral and the arrangement in (ii) has apparently higher symmetry than the original arrangement (i).

The transition from (i) to (ii) is continuous, that is, there is a gradual change of the two axes $a$ and $b$ as the temperature increases with $\mathrm{C}_{21} \mathrm{H}_{44}$ and $\mathrm{C}_{23} \mathrm{H}_{48}$. From $\mathrm{C}_{24} \mathrm{H}_{50}$ onwards all the samples investigated show a sudden transition at about $5^{\circ}$ to $10^{\circ} \mathrm{C}$. below the melting point of the individual substance. The arrangement after this transition has taken place is still of the same type as (i) and approaches (ii) gradually as the temperature gets near the melting point. These transitions are incidentally those for which Garner and his collaborators have measured the heat of transition. ${ }^{2}$

The change from a less symmetrical arrangement at low temperature to another of higher symmetry at high tomperature is not difficult to visualise. The moment of inertia of the chain molecule relative to its axis is comparatively small, and oscillations of large amplitude or complete rotations are to be expected at higher temperatures. Such oscillations obviously tend to produce fields of force round the chain axes which, when averaged, show radial symmetry and produce the simple close packed structure found in the present experiments.

The observations show that too high a symmetry may be observed in a crystal if the temperature at which the measurements are made is above a certain limit. A similar case for a crystal of amylammonium chloride has recently been discussed by Pauling. ${ }^{3}$

Davy Faraday Laboratory

Alex. Müller.

Royal Institution,

London, W.1,

Feb. 24 .

1 Proc. Roy. Soc., A, 127, 417; 1931

J. Chem. Soc., p. $1533 ; 1931$

${ }^{3}$ Annual Survey of American Chem., 15, 118-125.

\section{Ovis astore, a Three-Goated Sheep}

IN Prof. Barker's description of the sheep from Astore (Gilgit), ${ }^{1}$ several points which bear upon his subsequent discussion of the development of the fleece of domesticated sheep are not clear. For example, it would be of great interest to know (1) whether the individual animal described is typical of many in that locality, that is, of a local variety or "breed' or is a crossbred, and (2) the conditions of husbandry under which the animal had been maintained while the coat was grown, since changes of husbandry, including nutrition, can give rise to marked changes in thickness of the fibres throughout their lengths.

The difficulties encountered in endeavours to relate the coat constitutions of domesticated sheep with a two-coated primitive type are mentioned by Prof. Barker, but when questions other than those of fibre structure are introduced, the situation becomes more complicated, since a classification of the constituent fibres into two groups is no longer possible. Crew, ${ }^{2}$ while insisting upon the two main types of hair and wool, distinguished three groups of hairs when classed according to length and colour in the summer coat of $O$. ammon poli. The coat of domesticated sheep exhibits two types of fibre-kemp and wool-and Fraser Roberts has shown that the distinction between these can best be made according to period and duration of growth; the 'heterotypes' of Duerden are fibres which exhibit kemp-like structures for a part of the length. Again, in the normal coat of the Scotch Blackface breed, the so-called 'double-coat' is really a triple coat, since the obvious groups of fibres are (1) kemp, (2) coarse, long fibres, and (3) fine, under-coat fibres.

Fraser Roberts, in a series of papers on "Colour Inheritance in Sheep ", 3 discusses the various regional differences in coat pigmentation and describes the types of pigmentation found in individual fibres in the coats of primitive forms; if fibre pigmentation is included, the possible classification of coat fibres is greatly extended. In the Suffolk breed occur kemps, coarse wool fibres, and fine wool fibres, each of which may be, at least, wholly pigmented, non-pigmented, banded, or parti-coloured; and even in the adult fleeces of this breed there appear to be different thresholds of pigment inclusions for the various types of fibre, it having been noted, for exarnple, that the kemps are more likely to be pigmented than are the neighbouring wool fibres growing during the same period. ${ }^{4}$ Further, in the Merino breed, with no coat colour pattern, individuals which show 'banded' fleeces are not uncommon, and Boyd ${ }^{5}$ recognised four main fibre types which contributed to the gross appearance of these fleeces, with, in one of four Merino fleeces analysed, an additional fifth type.

As Prof. Barker suggests, the degree or time of shedding of the fibres in the sheep described may throw some light upon its general coat constitution, but it may be questioned whether there is sufficient evidence

No. 3255, VoL. 129] 